\title{
The effect of Blastocystis sp. and Dientamoeba fragilis on psychological symptom severity in a sample of clinically diverse males and females
}

\author{
Michael Ganci $^{1}$ D $\cdot$ Henry Butt ${ }^{1,2,3} \cdot$ Jean Tyrrell $^{2} \cdot$ Emra Suleyman $^{1} \cdot$ Michelle Ball $^{1}$
}

Accepted: 31 March 2021 / Published online: 22 April 2021

(C) The Author(s) 2021

\begin{abstract}
Health outcomes associated with Blastocystis sp. and Dientamoeba fragilis are disparate and controversial, ranging from health benefits, to years of asymptomatic carriage, through to severe illness. Evidence that Blastocystis sp. and D. fragilis are commensal members of the gut microbiota is growing. Despite this, little to no research exists investigating the potential effect of these protozoa on psychological symptom expression. As such, the aim of this retrospective cross-sectional study was to be the first to investigate the effect of protozoan carriage on severity of Depressive, Neurocognitive, Stress and Anxiety, and Sleep and Fatigue symptoms, and whether this effect changes as a function of sex. The prevalence of $D$. fragilis was significantly higher in females compared to males, however there were no sex differences in prevalence for Blastocystis sp. (data used in the current study contained ST1, ST3, and Blastocystis ST unspecified) or co-carriage of the two. Females reported significantly more severe symptoms across all four psychological domains compared to males. There was no significant interaction between sex and Blastocystis sp. carriage on psychological symptom severity, and no significant main effect of Blastocystis sp. on symptom severity compared to those who tested negative for protozoa. When investigating the sexes separately, there was no effect of protozoan carriage on psychological symptom expression in either males or females. These findings add weight to the argument that Blastocystis sp. and D. fragilis are not necessarily pathogenic and are likely to be part of a diverse gut (which is typically associated with better health outcomes). Further research is required given that protozoan members of the gut microbiota have been largely ignored in brain-gut-microbiota axis research.
\end{abstract}

Keywords Blastocystis $\cdot$ Dientamoeba fragilis $\cdot$ Gut-brain axis $\cdot$ Psychological symptoms

\section{Introduction}

Research into the brain-gut-microbiota axis (BGMA) has uncovered multidirectional relationships between the microorganisms residing in the gut and the development and functioning of the central nervous system (e.g. El Aidy et al., 2016; Rea et al., 2016). Alterations in gut microbiota have been associated with mood (Jiang et al., 2015) and developmental disorders (Li et al., 2017) as well as neurodegenerative (Vogt

Michael Ganci

Michael.ganci1@live.vu.edu.au

1 Psychology Department, Institute for Health and Sport, Victoria University, Melbourne, PO Box 14428, Melbourne, VIC 8001, Australia

2 Bioscreen Yarraville (Aust) Pty Ltd., Melbourne, VIC, Australia

3 Melbourne University, Parkville, VIC 3010, Australia et al., 2017) and neuroimmune conditions (Giloteaux et al., 2016). However, the vast majority of BGMA research has focused solely on the bacterial component of this ecosystem. While research has been undertaken investigating associations between eukaryotic protozoa and gastrointestinal (GI) disorders such as inflammatory bowel disease (IBD) and irritable bowel syndrome (IBS) as well as other GI symptoms, there is limited research into the influence of protozoa on psychological functioning. Further still, there does not appear to be any research to date which has focused specifically on Blastocystis sp. and Dientamoeba fragilis, two commonly detected intestinal protozoa (e.g. Aykur et al., 2019; Tito et al., 2019).

A growing body of evidence suggests that protozoa Blastocystis sp. and D. fragilis are commensal members of the gut microbiota that have coevolved with their human host (e.g. Beghini et al., 2017; Chabé et al., 2017; Scanlan et al., 2014). A number of studies have demonstrated either no differences in the prevalence of Blastocystis sp. and/or D. fragilis in asymptomatic groups compared to those with IBS, IBD, or other GI symptoms 
(e.g. Jokelainen et al., 2017; Seyer et al., 2017) or even a higher prevalence of these protozoa in asymptomatic groups (e.g. Beiromvand et al., 2017; Brands et al., 2019; Holtman et al., 2017; Rossen et al., 2015; Tito et al., 2019). One possible explanation for the protective nature of these protozoa is the association between carriage of Blastocystis sp. and/or D. fragilis and higher gut bacterial richness and diversity (Audebert et al., 2016; Krogsgaard et al., 2018; Tito et al., 2019) which are indicators of a healthy microbiota (e.g. Bruce-Keller et al., 2018; Lloyd-Price et al., 2016; Rinninella et al., 2019; Valdes et al., 2018).

From an evolutionary perspective, intestinal microorganisms that have coevolved with their human host, including protozoa, may have immunomodulating effects (Chudnovskiy et al., 2016; Deng et al., 2021; Maizels, 2009). The 'old friends' hypothesis (Rook \& Brunet, 2005) proposes that lower exposure to immunoregulating intestinal microorganisms may be related to the higher prevalence of chronic and inflammatory diseases seen today (Chabé et al., 2017; Rook, 2013; 2014). This is supported by the higher incidence of inflammatory and allergic diseases and lower prevalence of intestinal protozoa seen in industrialised countries compared to non-industrialised countries that maintain a more traditional lifestyle, which have a higher prevalence of protozoa and lower incidence of inflammatory and allergic disease (Chabé et al., 2017; Lokmer et al., 2019). However, coevolution can alternatively lead to an evolutionary arms race; a cycle of adaptations and counter-adaptations between host and protozoan (Dawkins \& Krebs, 1979; Laanto et al., 2017). In contrast to the 'old friends' hypothesis which proposes immunological benefits for the human host, immune responses induced by Blastocystis sp. may instead result in increased symptomatology (El-Zawawy et al., 2020). Currently, knowledge regarding the immune response to D. fragilis remains lacking (Yadav et al., 2020), contributing to its label as a neglected protozoan (e.g. AlHindi \& Abu-Shammala, 2013; Garcia, 2016) and to the uncertainty over its pathogenicity.

As such, controversy remains over the potential pathogenicity of these protozoa (e.g. Garcia, 2016; Lepczyńska et al., 2017) with other studies finding an increased prevalence in groups with GI symptoms (Crotti \& D'Annibale, 2007; Kesuma et al., 2019; Norberg et al., 2003). These inconsistencies may be due to several factors which could alter the pathogenicity of these otherwise commensal organisms including the bacterial component of the gut microbiota (Burgess et al., 2017), protozoan load (Pavanelli et al., 2015), duration of carriage (Kaneda et al., 2000; Lukeš et al., 2015), and the sex and immune functioning of the host (Chandramathi et al., 2012; Nourrisson et al., 2014). Specific protozoan subtypes (STs) have also been associated with varying symptom outcomes (Bart et al., 2013; Zulfa et al., 2017). Blastocystis sp. is one such example with 17 subtypes (STs) identified, of which STs $1-9$ and ST12 have been identified in humans (Jiménez et al., 2019; Stensvold \& Clark, 2016; Tito et al., 2019). Chandramathi et al. (2014) also demonstrated that stress can exacerbate the pathogenic potential of Blastocystis sp.
Despite the continued debate about the pathogenicity of Blastocystis sp. and D. fragilis, typical treatments for these protozoa include administering antimicrobial agents (such as metronidazole and trimethoprim-sulfamethoxazole among several others; Coyle et al., 2011; Nagata et al., 2012). However, these treatments have potentially serious side effects such as confusion and headaches, nausea, vomiting, and may disrupt the delicately balanced microbial ecosystem in the gut (Ho \& Juurlink, 2011; Weir \& Le, 2020). Treatment for asymptomatic individuals is therefore not recommended (Coyle et al., 2011).

Comparatively, psychological outcomes of Blastocystis sp. and/or $D$. fragilis carriage have received little to no attention, particularly in humans. There is only very limited evidence of a possible link between these protozoa and fatigue (Johnson et al., 2004; Norberg et al., 2003; Qadri et al., 1989). However, this evidence is weak as it typically comes from case reports (Butler, 1996; Dunwell, 2013) or studies where other possible causes, such as IBS, were not ruled out (Norberg et al., 2003). Conversely, Holtman et al. (2017) found an association between the absence of $D$. fragilis and fatigue in children, however they offered no viable explanation for this result.

In regard to psychological symptom expression, research suggests that there are differences between males and females, with females tending to report more sleep problems (Boccabella \& Malouf, 2017) and have a greater risk of anxiety and depression (Altemus et al., 2014). It is suggested that sex hormones interact with stress hormones in a way that makes females more vulnerable to stress-related disorders (Solomon \& Herman, 2009). Additionally, Hall and Steiner (2013) explain females' greater susceptibility to various psychopathologies by suggesting that sex hormones, particularly fluctuations in estrogens, may interact with the serotonergic system. Sex differences in immunoregulatory function (Sorge \& Totsch, 2017) and symptom expression and reporting (Barsky et al., 2001; MacLean et al., 2010) must also be considered in relation to the effect of protozoan carriage on symptom expression (e.g. Maeng \& Milad, 2015; Mulak et al., 2014; Seney \& Sibille, 2014; Sorge \& Strath, 2018). This is, due to the immunoenhancing effect of estrogen, and the immunosuppressive effects of testosterone (Kovats, 2015; Taneja, 2018), which may influence susceptibility to, and the consequential effects of, intestinal protozoa. In addition to sex differences in symptom expression, the microgenderome is a paradigm highlighting sex specific host-microbiota interactions (Flak et al., 2013; Markle et al., 2013). Research focusing on the bacterial component of the microbiota has provided support for this paradigm (e.g. Vemuri et al., 2019; Wallis et al., 2016). Studies investigating protozoan carriage have provided further support for the microgenderome with different health outcomes reported between males and females (Klein, 2004; Marriott \& HuetHudson, 2006; Salehi et al., 2021; Zuk, 2009). Nourrisson et al. (2014) found that Blastocystis sp. was associated with 
IBS only in males, and that carriage was also associated with a reduction of Bifidobacteria and Faecalibacterium prausnitzii in male control group participants. Generally, protozoan carriage is more prevalent in males (Abu-Madi et al., 2016; Klein, 2004), however there are some exceptions, such as D. fragilis, which trends towards higher prevalence in females (Barratt et al., 2011; Crotti \& D'Annibale, 2007; Grendon et al., 1995; Röser et al., 2013). However, there are inconsistencies within the literature with some studies showing relatively equal prevalence of $D$. fragilis in males and females (Al-Hindi \& Abu-Shammala, 2013; Hamidi et al., 2018; Stark et al., 2010). As such, sex differences in susceptibility to, carriage of, and health outcomes associated with protozoa remain poorly understood and more research is needed.

While the majority of studies investigating intestinal protozoa Blastocystis sp. and D. fragilis have thus far centred their investigation on GI symptoms, this study served to take an essential first step to fill a gap in the literature investigating the influence of these two protozoa on psychological symptom severity across Depressive, Neurocognitive, Stress and Anxiety, and Sleep and Fatigue symptom domains. The aim of the current study was to determine whether there is an effect of Blastocystis sp. and/or D. fragilis carriage on self-reported psychological symptom severity, and whether this effect changes as a function of sex. Given that this is the first study to the authors' knowledge which has investigated the influence of these specific protozoa on psychological symptom outcomes, this study is exploratory, and as such, what is to be expected is somewhat uncertain. However, given the increasing evidence that Blastocystis sp. and D. fragilis are part of a healthy gut (e.g. Chabé et al., 2017) the following hypotheses are put forward. Firstly, it was hypothesised that carriage of Blastocystis sp. and/or D. fragilis would not have a detrimental effect on symptom severity. Secondly, it was hypothesised that females would self-report more severe symptoms compared to males in all four psychological domains. Finally, in line with research in support of the microgenderome, it was hypothesised that the effect of protozoan carriage on symptom expression would differ as a function of sex.

\section{Materials and Methods}

\section{Study Participants}

Data analysed in the current study was taken from a larger retrospective database of 4610 clinically diverse adult (18 years or above) patients who were referred to Bioscreen, a Melbourne based laboratory specialising in faecal microbial analysis (FMA), between February 2013 and June 2015. The subsection of the sample used in the current study were those whose stool samples were tested for intestinal protozoa $(n=$
988). Stool samples were submitted for analysis as part of the investigatory process for intestinal dysbiosis however, the specific diagnostic status of patients were not identified. As such, this is a cross-sectional study of a broad range of potentially clinical and non-clinical presentations. Ethics approval for the current study was obtained from the Victoria University Human Research Ethics Committee (HRE16071).

\section{Inclusion and Exclusion Criteria}

From the larger database, inclusion criteria for the current study was that two-step polymerase chain reaction (PCR) testing was conducted to indicate the presence or absence of intestinal protozoa, that patients completed the Bioscreen patient questionnaire (BPQ), and that patients also provided consent for their information to be used in future research. Patients were excluded from the current study if PCR testing, BPQ data, or consent were not provided, or if their stool sample was unsuitable for testing as defined by quality control guidelines. A further exclusion criterion was if patients tested positive for intestinal protozoa other than Blastocystis sp. or D. fragilis (including Cryptosporidium and Giardia; $n=9$ ) or any other known pathogens (e.g. Clostridium difficile). No further information was available to exclude other possible extraneous of confounding variables.

Overall, 979 patients met the criteria for inclusion in the current study. Available demographic information for the sample is presented in Table 1 .

\section{Sample Collection and Parasite PCR Assay}

Stool samples were collected by patients according to directions provided in a Bioscreen FMA kit which was posted to their home. Patients stool samples were kept anaerobically and transported via express post (typically next day delivery) to the laboratory in cool conditions (below $12^{\circ} \mathrm{C}$ ). Patients were instructed to complete the BPQ within temporal proximity to faecal sample collection and return this with the kit. On receipt, samples were kept at $-20{ }^{\circ} \mathrm{C}$ until tested (within three days). Samples were subjected to internal quality assurance and were rejected if they had been inaccurately collected, transported, refrigerated, or delayed in transit.

Table 1 Sample demographic information

\begin{tabular}{llll}
\hline Sex & $n(\%)$ & Age range & Mean age \\
\hline Male & $258(26.4)$ & $18-82$ & $43.582(S D=14.013)$ \\
Female & $721(73.6)$ & $18-86$ & $42.796(S D=13.124)$ \\
Total & 979 & $18-86$ & $43.003(S D=13.361)$ \\
\hline
\end{tabular}


On the day of testing, faecal samples were thawed at room temperature. A custom-made 7-well target panel was used to screen for Blastocystis sp., Blastocystis subtype (ST) 1 and 3, D. fragilis, Entamoeba histolytica, Cryptosporidium, and Giardia (Ausdiagnostics Pty Ltd., Sydney, Australia). DNA extractions were carried out using a QIAamp DNA Stool Mini Kit and QIAcube (Qiagen, Melbourne, Australia). In short, $200 \mathrm{mg}$ thawed samples were homogenised using inhibitEX buffer (Qiagen) and centrifuged. A Two Step Multiplex PCR was carried out as per the manufacturer's instructions (Ausdiagnostics). Amplification using primers homologous to all targets in the panel were used. Gene targets for each assay can be found in Table 2. Real-time PCR was automated by the Easy-Plex system (AusDiagnostics). The Ultra-Plex analyser (Ausdiagnostics) was used for DNA amplification.

\section{Faecal Microbial Analysis (FMA)}

The FMA process used in the current study was identical to that performed in previous studies conducted by Coulson et al. (2013) and Wallis et al. (2016). In brief, culture methods, on a variety of media (previously dried Columbia horse blood agar (Oxid, Thermo Fisher, Australia), chromogenic medium (Oxid), colistin and nalidixic acid blood selective agar (Oxid), and chloramphenicol-gentamicin selective Sabouraud agar), were used to perform bacterial counts within $48 \mathrm{~h}$ of sample collection. Specifically, Matrix Assisted Laser Absorption and Ionisation Time of Flight Mass Spectrometry (MALDI-TOF-MS) using a proprietary peptide database (MALDI Biotyper Bruker Daltonics, Bremen, Germany) was utilised for identification.

\section{Bioscreen Patient Questionnaire (BPQ)}

The BPQ is an 88-item questionnaire developed by Bioscreen administered to patients as part of Bioscreen's standard procedure. Items on the BPQ are similar in nature to other symptom checklists which relate to diverse symptomatology and patients are asked to report the frequency (over the past 12 months) and severity (over the past seven days) of their

Table 2 Assay gene targets

\begin{tabular}{ll}
\hline Assay & Gene target \\
\hline E. histolytica & Surface antigen ariel 1 \\
D. fragilis & $18 \mathrm{~S}$ \\
Cryptosporidium & Oocyte wall protein \\
Giardia & $18 \mathrm{~S}$ \\
Blastocystis ST 1 & Small subunit ribosomal RNA \\
Blastocystis ST 3 & Small subunit ribosomal RNA \\
BlastE1-a & Elongation factor 1-alpha \\
\hline
\end{tabular}

symptoms on a five-point Likert scale ranging from zero to four, with higher scores indicating higher ratings of frequency/severity. For the purposes of the current study, only symptom severity over the past seven days was assessed, given the underlying assumption of a temporal relationship between protozoan carriage and symptom expression. This is the first study to use mathematically derived symptom factors from the BPQ. The methods used as well as sub-scale reliabilities are outlined in the data handling and statistical design section.

\section{Data Handling and Statistical Design}

\section{Deriving Symptom Domains from the BPQ}

Symptom domains were derived mathematically by subjecting the BPQ to factor analysis using the maximum likelihood (ML) method of extraction in the larger sample from which the current sample was drawn (see Online Resource 1). The factor analysis revealed that of the 88 original items, 53 items loaded onto 10 distinct factors, with minimal cross loadings. These 10 factors covered Depressive (6 items; Cronbach's $\alpha=0.894)$, Pain (6 items; Cronbach's $\alpha=0.849$ ), Neurocognitive ( 8 items; Cronbach's $\alpha=0.938$ ), GI (5 items; Cronbach's $\alpha=0.782$ ), Stress and Anxiety (9 items; Cronbach's $\alpha=0.874$ ), Sleep and Fatigue (6 items; Cronbach's $\alpha=0.853$ ), Exertion (4 items; Cronbach's $\alpha=$ 0.820 ), Urination (3 items; Cronbach's $\alpha=0.684$ ), Neck and Shoulder pain ( 2 items; Cronbach's $\alpha=0.847$ ), and Immunity (4 items; Cronbach's $\alpha=0.652$ ) symptom domains. The specific items that loaded onto each symptom domain factor can be found in Table 3 in Online Resource 1. For the purposes of the current study, only the psychological domains (Depressive, Neurocognitive, Stress and Anxiety, and Sleep and Fatigue symptoms) were used in all further analyses.

\section{Combining Blastocystis Subtypes (STs)}

Prior to any further analysis, a decision was made to combine patients who tested positive for Blastocystis ST unspecified, Blastocystis ST1, Blastocystis ST3, and co-carriage of Blastocystis ST1 and ST3 into a single variable, allowing for the analysis of Blastocystis sp. at the genus level. While it would be ideal to compare the effect of the various Blastocystis STs, this is not possible given the current retrospective database for three main reasons. The first is that it was only specified whether a patient had Blastocystis ST1, Blastocystis ST3 (or co-carried both ST1 and ST3), or Blastocystis ST unspecified with no information being available regarding the other eight Blastocystis STs that have been identified in humans. Secondly, for those who tested positive for Blastocystis ST unspecified, it cannot be concluded with any degree of certainty which of the 10 Blastocystis ST they potentially carried. Finally, the percentage of patients who 
Table 3 Blasocystis and D. fragilis prevalence $(N=979)$

\begin{tabular}{|c|c|c|c|c|c|c|}
\hline & \multicolumn{2}{|c|}{ Males $(n=258)$} & \multicolumn{2}{|c|}{ Females $(n=721)$} & \multicolumn{2}{|c|}{ Total $(n=979)$} \\
\hline & $n$ & $\begin{array}{l}\text { Mean age in } \\
\text { years }(S D)\end{array}$ & $n$ & $\begin{array}{l}\text { Mean age in } \\
\text { years }(S D)\end{array}$ & $n$ & $\begin{array}{l}\text { Mean age in } \\
\text { years }(S D)\end{array}$ \\
\hline Protozoan negative & 161 & $43.90(14.37)$ & 402 & $43.20(13.73)$ & 563 & $43.40(13.90)$ \\
\hline Blastocystis sp. & 75 & $43.25(13.39)$ & 199 & $43.06(13.16)$ & 274 & $43.11(13.20)$ \\
\hline D. fragilis & 10 & $44.46(13.14)$ & 59 & $38.99(9.97)$ & 69 & $39.78(10.55)$ \\
\hline Co-carriage & 12 & $40.62(14.97)$ & 61 & $42.95(11.15)$ & 73 & 42.57 (11.77) \\
\hline Total & 258 & $43.58(14.01)$ & 721 & $42.80(13.12)$ & 979 & $43.00(13.36)$ \\
\hline
\end{tabular}

tested positive for any Blastocystis ST $(n=274)$ was $67.2 \%$ for Blastocystis ST unspecified ( $n=184), 13.1 \%$ for ST1 $(n=$ $36), 16.8 \%$ for ST3 ( $n=46)$, and $2.9 \%$ for co-carriage of ST1 and ST3 $(n=8)$. These discrepancies in sample size are further exacerbated when considering the comparison to patients who tested negative to protozoan carriage $(n=563)$, and again exacerbated when separating for sex. It is believed that this decision would allow for the most meaningful analysis, given the data that was available.

As a safeguard to ensure that this would not introduce undue bias into the results, a $3 \times 2$ MANOVA was conducted to determine if there was a significant difference in the endorsement of psychological symptom severity between the Blastocystis STs (Blastocystis ST unspecified, Blastocystis ST1, and Blastocystis ST3) and whether this effect varied as a function of sex (male or female). The MANOVA demonstrated no significant interaction between Blastocystis STs and sex, and also showed no significant main effect for Blastocystis STs on psychological symptom severity. These findings support the decision to collapse the Blastocystis STs into a single variable to allow for investigation of Blastocystis $\mathrm{sp}$. at the genus level in subsequent analyses.

\section{Independent and Dependent Variables}

The independent variable (IV), protozoan carriage status, was coded as having four levels (protozoan negative, Blastocystis sp. positive, $D$. fragilis positive, or co-carriage of Blasotcystis together with $D$. fragilis). Due to the low prevalence of $D$. fragilis and co-carriage in males (as seen in Table 3), these groups were excluded from further statistical analysis. Therefore, when males were included in analyses, only two levels of the IV were considered (protozoan negative or Blastocystis sp. positive). Factor scores for each of the four psychological symptom domains were calculated and used as the dependant variables (DVs) in three multivariate analyses (MANOVA). The first, was a $2 \times 2$ (Blastocystis $\mathrm{sp}$. [positive or negative] x sex [male or female]) independent measures MANOVA to determine the effect of Blastocystis sp. carriage on psychological symptom severity, and whether this effect changed as a function of sex. When separating for sex, differences in psychological symptomatology between males who tested negative for protozoa and those who tested positive for Blastocystis sp. were analysed using a one-way independent measures MANOVA. For females, who had a sufficient sample size across all four levels of the IV, a separate one-way independent measures MANOVA was used to compare psychological symptomatology.

Chi-square test of independence was used determine whether there was an association between sex and protozoan carriage, with Cramer's V used to determine the effect size of this association (Akoglu, 2018; McHugh, 2013).

\section{Statistical Assumptions}

The assumptions of normality (skewness and kurtosis) and multivariate normality (using the Mahalnobis distance critical value appropriate for four DVs) were assessed and met. The assumption of homogeneity of variance was not violated for all following analyses. Moreover, the Box's M Test of Equality of Covariance Matrices demonstrated that the assumption of homogeneity of variance-covariance matrices was also met for all analyses according to Hubery and Petoskey's (2000) guideline of $p<.005$. Pearson's correlation coefficients in the moderate range demonstrated that multicollinearity did not exist between the four DVs. A linear relationship existed between each pair of DVs. No extreme outliers were detected. Finally, listwise deletion was used to deal with missing data. All analyses were interpreted using an alpha $(\alpha)$ of .05.

\section{Results}

\section{Protozoan Prevalence}

Information regarding the prevalence of Blastocystis sp. and D. fragilis is shown in Table 3.

Based on the protozoan distribution among males and females seen in Table 3, chi-square test for independence 
showed that there was a significant association between protozoan carriage and sex, $\chi^{2}(3, N=979)=10.305, p=.016$. The associated Cramer's V of .103 suggests that the effect size of this association is small to medium (Akoglu, 2018). Interpretation of the adjusted standardised residuals (Beasley \& Schumacker, 1995) demonstrated that the prevalence of $D$. fragilis was significantly higher in females compared to males $(p=.020)$. The prevalence of co-carriage was also significantly higher in females $(p=.046)$. There was no significant difference in the prevalence of Blastocystis sp. between males and females, nor was there a significant difference in the number of males and females who tested negative for either Blastocystis sp. or D. fragilis.

\section{Effect of Blastocystis Sp. on Psychological Symptom Expression as a Function of Sex}

Presented in Table 4 are the descriptive statistics relevant to the $2 \times 2$ MANOVA and the one-way MANOVA analysing symptom severity in males. Mean $(M)$ and standard deviation $(S D)$ symptom severity scores for males and females who tested negative for protozoan carriage and those who tested positive for Blastocystis sp. are presented for all four psychological symptom domains. Mean and standard deviation values for $D$. fragilis and co-carriage which relate only to females can be found in Table 4 .

A $2 \times 2$ MANOVA was run to determine whether there was a significant effect of Blastocystis sp. carriage on symptom severity compared to protozoan negative patients and whether this effect differed as a function of sex. The results showed no significant interaction between Blastocystis sp. carriage and sex on symptom severity. This demonstrates that the effect of Blastocystis sp. on psychological symptom severity is similar in males and females. Given the nonsignificant interaction, the main effects of sex and Blastocystis sp. carriage were considered. The results also demonstrated a non-significant main effect for Blastocystis sp. on symptom severity, meaning that irrespective of sex, there was no difference in psychological symptom severity between those who were protozoan negative and those who were Blastocystis sp. positive. However, there was a significant main effect of sex on symptom severity, Wilks' $\Lambda=.951$, $F(4,719)=9.182, p<.001, \eta_{p}^{2}=.049$, meaning that regardless of protozoan carriage status, there was a difference between males and females on psychological symptom severity. Interpretation at the univariate level revealed that females reported significantly more severe Depressive $(F[1,722]=$ 18.266, $\left.p<.001, \eta_{p}^{2}=.025\right)$, Neurocognitive $(F[1,722]=$ $\left.8.899, p=.003, \eta_{p}{ }^{2}=.012\right)$, Stress and Anxiety $(F[1,722]=$ 8.978, $\left.p=.003, \eta_{p}{ }^{2}=.012\right)$, and Sleep and Fatigue $(F[1$, $\left.722]=33.671, p<.001, \eta_{p}{ }^{2}=.045\right)$ symptoms compared to males. It should be noted that these effect sizes are small (partial eta squared values between .0099 and .0587; Cohen, 1969; Richardson, 2011), indicating that only a small amount of variance in psychological symptom severity is accounted for by sex alone.

\section{Effect of Protozoan Carriage within each Sex}

\section{Males}

A one-way independent measures MANOVA was run to determine whether there was a significant difference between males who tested negative for protozoan carriage, and those who were positive for Blastocystis sp. on their self-reported psychological symptom severity. As mentioned in the data

Table 4 Mean symptom severity scores for protozoan negative and Blastocystis sp. positive males and females

\begin{tabular}{|c|c|c|c|c|c|c|c|c|c|c|}
\hline \multirow[t]{2}{*}{ Symptom domain (possible range) } & \multirow[t]{2}{*}{ Protozoan status } & \multicolumn{3}{|c|}{ Males (M) } & \multicolumn{3}{|c|}{ Females $(\mathrm{F})$} & \multicolumn{3}{|c|}{ Total combined across $\mathrm{M}$ and $\mathrm{F}$} \\
\hline & & $n$ & $M$ & $S D$ & $n$ & $M$ & $S D$ & $n$ & $M$ & $S D$ \\
\hline \multirow[t]{3}{*}{ Depressive $(0-24)$} & Negative & 146 & 7.137 & 6.478 & 346 & 9.127 & 6.958 & 492 & 8.537 & 6.873 \\
\hline & Blastocystis sp. & 65 & 6.354 & 6.333 & 169 & 9.414 & 6.512 & 234 & 8.564 & 6.594 \\
\hline & Total & 211 & 6.896 & 6.429 & 515 & 9.221 & 6.810 & & & \\
\hline \multirow[t]{3}{*}{ Neurocognitive $(0-32)$} & Negative & 146 & 9.843 & 8.895 & 346 & 12.009 & 9.202 & 492 & 11.366 & 9.157 \\
\hline & Blastocystis sp. & 65 & 9.646 & 8.219 & 169 & 12.201 & 8.881 & 234 & 11.492 & 8.760 \\
\hline & Total & 211 & 9.782 & 8.673 & 515 & 12.072 & 9.090 & & & \\
\hline \multirow{3}{*}{$\begin{array}{l}\text { Stress/Anxiety } \\
(0-36)\end{array}$} & Negative & 146 & 8.096 & 8.138 & 346 & 10.254 & 8.212 & 492 & 9.614 & 8.241 \\
\hline & Blastocystis sp. & 65 & 7.939 & 7.925 & 169 & 10.107 & 8.312 & 234 & 9.504 & 8.241 \\
\hline & Total & 211 & 8.047 & 8.054 & 515 & 10.206 & 8.237 & & & \\
\hline \multirow{3}{*}{$\begin{array}{l}\text { Sleep/Fatigue } \\
(0-24)\end{array}$} & Negative & 146 & 7.952 & 5.715 & 346 & 10.104 & 4.924 & 492 & 9.465 & 5.259 \\
\hline & Blastocystis sp. & 65 & 6.631 & 5.030 & 169 & 9.722 & 5.040 & 234 & 8.863 & 5.215 \\
\hline & Total & 211 & 7.545 & 5.535 & 515 & 9.979 & 4.961 & & & \\
\hline
\end{tabular}


handling and statistical design section above, males who tested positive for $D$. fragilis or co-carriage of Blastocystis sp. and $D$. fragilis were excluded from analysis due to small sample size which precluded meaningful comparisons. Refer to Table 4 for descriptive information relating to symptom severity for protozoan negative and Blastocystis sp. positive males. The results demonstrated no significant difference between protozoan negative and Blastocystis sp. positive males with regard to their psychological symptom severity.

\section{Females}

Descriptive information relating to symptom severity in females can be found in Table 5 .

A final one-way independent measures MANOVA was run to determine whether there was a significant difference in selfreported psychological symptom severity between females who tested positive for Blastocystis sp., D. fragilis, or cocarriage compared to those who tested negative for protozoa. Descriptive information relating to symptom severity in females can be found in Table 5. Results demonstrated that protozoan carriage status had no significant effect on psychological symptom severity.

\section{Interaction between Protozoa and Bacterial Composition (Bacteroidetes and Firmicutes)}

Additional analyses were conducted to determine whether bacteria (at the phylum level) moderated the relationship between protozoan carriage and psychological symptom severity. The Firmicutes and Bacteroidetes phyla were selected as moderating variables as these two phyla provide a general snapshot of the bacterial component of the gut microbiota. Several moderation analyses were conducted through the PROCESS macro tool for SPSS (Hayes, 2017). First, the protozoan variable (which had four levels) was dummy coded for use in regression analysis (Cohen et al., 2013). The amounts of Firmicutes and Bacteroidetes were first log-transformed and then mean-centred through the PROCESS macro tool (Hayes, 2017). The results revealed that the level of either Firmicutes or Bacteroidetes did not moderate the relationship between protozoan carriage and psychological symptom severity across any of the four psychological symptom domains for males or females (data not shown). As such, the effect of protozoan carriage was not altered by either phyla.

\section{Discussion}

This retrospective study contributes to the mounting evidence that carriage of Blastocystis sp. and/or D. fragilis do not have a negative effect on symptom expression and that these protozoa are likely to be commensal members of the gut microbiota (e.g. Beghini et al., 2017; Jokelainen et al., 2017; Lukeš et al., 2015). These results need to be interpreted in the context of limitations relating to the retrospective study design and methodological challenges which face all studies investigating protozoa and the BGMA more widely which will be discussed herein. Despite these limitations the current study has extended the scope of symptoms investigated with regard to Blastocystis sp. and D. fragilis carriage beyond GI alone, and is the first to investigate psychological symptoms (Depressive, Neurocognitive, Stress and Anxiety, and Sleep and Fatigue) that have previously received no attention. These
Table 5 Mean symptom severity scores relating to protozoan carriage status in females

\begin{tabular}{lllll}
\hline Symptom domain(possible range) & Protozoan status & $n$ & $M$ & $S D$ \\
\hline Depressive(0-24) & Negative & 346 & 9.127 & 6.958 \\
& Blastocystis sp. & 169 & 9.414 & 6.512 \\
& D. fragilis & 53 & 7.528 & 6.259 \\
& Co-carriage & 56 & 9.071 & 6.907 \\
Neurocognitive & Negative & 346 & 12.009 & 9.202 \\
(0-32) & Blastocystis sp. & 169 & 12.201 & 8.881 \\
& D. fragilis & 53 & 10.981 & 7.824 \\
& Co-carriage & 56 & 11.321 & 8.908 \\
Stress/Anxiety & Negative & 346 & 10.254 & 8.212 \\
$(0-36)$ & Blastocystis sp. & 169 & 10.107 & 8.312 \\
& D. fragilis & 53 & 8.604 & 6.698 \\
Sleep/Fatigue & Co-carriage & 56 & 9.304 & 8.228 \\
$(0-24)$ & Negative & 346 & 10.104 & 4.924 \\
& Blastocystis sp. & 169 & 9.722 & 5.040 \\
& D. fragilis & 53 & 8.396 & 5.436 \\
& Co-carriage & 56 & 8.375 & 5.065 \\
\hline
\end{tabular}


findings inform future research into the BGMA and echo previous authors who highlight the need for further research into Blastocystis sp. and D. fragilis.

The hypothesis that carriage of Blastocystis sp. and/or $D$. fragilis would not have a detrimental effect on symptom severity was supported. It was found that for males, there was no difference in self-reported psychological symptom severity between those who tested negative for protozoan carriage and those who tested positive for Blastocystis sp. Due to sample size restrictions, the effect of $D$. fragilis and co-carriage of Blastocystis sp. and D. fragilis was unable to be analysed in males. Females also showed no differences in self-reported psychological symptom severity between those who were protozoan negative, or those who tested positive for Blastocystis sp., D. fragilis, or co-carriage of the two. While this study is the first to explore the effect of these specific protozoa on psychological symptom severity, the current findings reflect previous research that demonstrates Blastocystis sp. and D. fragilis not to be harmful in relation to GI symptoms (e.g. Jokelainen et al., 2017; Krogsgaard et al., 2015; Scanlan et al., 2014; Tito et al., 2019). There is a growing consensus that these intestinal protozoa are common members of a healthy gut microbiota (e.g. Audebert et al., 2016; Krogsgaard et al., 2018), which is in line with the 'old friends' hypothesis (Rook $\&$ Brunet, 2005) and the co-evolution of these protozoa with their human host (Chabé et al., 2017). Taking an evolutionary perspective, common protozoa that have coevolved with their human host, such as Blastocystis sp. and D. fragilis, may also contribute to a healthy gut to ensure their own fitness and survival. Tsaousis et al. (2018) suggest that the dysbiotic gut of IBS patients is an unsuitable habitat for the anaerobic Blastocystis sp., meaning that for its own survival, it would benefit from supporting a healthy gut. The same may also hold true for the anaerobic D. fragilis (Dunwell, 2013), although this has not been specifically tested. As such, it is suggested that the commensalism exhibited in studies focusing on GI symptoms is reflected systemically.

Also as hypothesised, females reported more severe psychological symptoms across all four domains (Depressive, Neurocognitive, Stress and Anxiety, and Sleep and Fatigue). This is consistent with a large body of previous literature that suggests females tend to experience and/or report both physiological and psychological symptoms more than males (e.g. Mulak et al., 2014; Seney \& Sibille, 2014; Sorge \& Strath, 2018). This is believed to be a product of biological sex differences which include interactions between sex hormones (particularly estrogens) both stress hormones (Solomon \& Herman, 2009) and the serotonergic system (Hall \& Steiner, 2013). Socialised gender expectations are also believed to play a role in greater reporting of various psychological symptoms in females (Barsky et al., 2001). However, the small effect sizes found in the current study suggest that sex itself only explains a small amount of the variance in self-reported psychological symptom severity and that there are likely to be other contributing factors of greater relevance or importance.

Finally, the hypothesis that the effect of protozoan carriage on self-reported symptom expression changes as a function of sex was not supported. However, as the sample of males who tested positive for $D$. fragilis and co-carriage was insufficient for meaningful comparisons to be drawn, the analysis was limited to the interaction between sex and Blastocystis sp. The results demonstrated that Blastocystis sp. had a similar effect on self-reported psychological symptom expression in males and females and as such does not offer support for the microgenderome. This finding contrasts those of Nourrisson et al. (2014) who found Blastocystis sp. to be related to IBS and a reduction of protective anti-inflammatory bacteria only in males. However, this result should not be taken as evidence against the microgenderome more broadly, as these findings relate to Blastocystis sp. in exclusion of all other factors (including other intestinal microbiota) and as such results may differ when considering the protozoan in the context of these other factors. With regard to $D$. fragilis, the disparity in prevalence between males and females makes it highly possible that sex hormones may interact with the protozoan, and possibly other intestinal protozoa. Further research is needed to explore these possibilities, as this may have important implications for faecal microbiota transplantation (FMT) donor selection and screening procedures.

In line with previous findings by those such as Crotti and D'Annibale (2007) and Grendon et al. (1995), the main point of difference between the sexes was the higher prevalence of $D$. fragilis in females. The higher prevalence of $D$. fragilis in females is despite the fact that, in general, males are more susceptible to protozoan and parasitic infections as a result of immunosuppressive androgens, while immunoenhancing oestrogen is proposed to contribute to resistance in females (Klein, 2004). However, the argument of immune resistance may not apply to Blastocystis sp. and D. fragilis which further supports their role as commensal organisms. If considering Blastocystis sp. and D. fragilis to be commensal members of the gut microbiota that have co-evolved with their human host, the immune system should not respond in the same way as it would to pathogenic protozoa and parasitic infections. This would explain the similar prevalence of Blastocystis sp. found in males and females, but still does not explain the increased prevalence of $D$. fragilis in females. Instead, this may be explained by cultural and behavioural differences between males and females (Barratt et al., 2011). For example, females tend to have close and frequent contact with young children (Rhoads \& Rhoads, 2012) which may make them more susceptible. Supporting this argument, Röser et al. (2013) found that only females of parental age had a significantly higher prevalence of $D$. fragilis, while Menendez Fernandez-Miranda et al. (2018) found the prevalence of $D$. fragilis to be associated with having children in the 
home. Beyond parenthood, this may also extend to those who work in jobs such as child care, which tends to be dominated by females (van Polanen et al., 2017).

Given that the gut microbiota is a highly complex ecosystem in which protozoan members cohabitate and interact with other constituents such as bacteria (e.g. Burgess et al., 2017; Leung et al., 2018), it is difficult to comment exclusively on the effect of protozoa on symptom severity. As such, moderation analyses were conducted to determine whether bacteria (at the phylum level) moderated the relationship between protozoan carriage and psychological symptom severity. The Firmicutes and Bacteroidetes phyla were selected as moderating variables as these two phyla alone represent approximately $90 \%$ of gut microbiota (e.g. Rinninella et al., 2019). While phylum level data lacks the specificity of genus or species level data, the ratio of Firmicutes to Bacteroidetes continues to be used as a general measure of bacterial composition (e.g. Durk et al., 2019; Stojanov et al., 2020; Vaiserman et al., 2020). The results of the current study revealed that the level of either Firmicutes or Bacteroidetes did not moderate the relationship between protozoan carriage and psychological symptom severity across any of the four psychological symptom domains for males or females. This suggests that the effect of protozoan carriage was not altered by either phyla. It was beyond the scope of this paper to investigate interactions between protozoa and bacteria at the more specific genera and species levels, however this should be addressed in future studies.

The limitations of the current study are mainly related to the restricted characterisation of the sample given its retrospective and cross-sectional nature. Useful information regarding protozoan load, duration of carriage, and formal diagnostic criteria of patients were not collected, and therefore unknown. Additionally, lifestyle factors such as diet (and others), and medication use which are known to influence overall gut ecology were not available. Not having this information available made it impossible to control for these factors or to analyse their potentially important interactions with Blastocystis sp. and/or D. fragilis. Additionally, due to the retrospective and cross-sectional nature of the data collection, determining the possible mechanisms of action of these protozoa was not possible. The retrospective data also limited analysis due to the cross-sectional observance of intestinal protozoa within the sample. In line with previous descriptions (see Barratt et al., 2011) D. fragilis was far less common in males in the current study which did not allow for meaningful comparisons between groups, or in fact within the male sample at all. One contributing factor to this is the highly variable day to day shedding of the protozoan requiring multiple sampling and analysis of freshly passed stool, potentially meaning that the presence of $D$. fragilis is generally underestimated in cross sectional research (Peek et al., 2004; Stark et al., 2016). With regard to Blastocystis sp., although 10 subtypes have been identified in humans, only ST1 and ST3 were distinguished from Blastocystis ST unspecified. Therefore, it was unknown which specific Blastocystis STs the majority of those who tested positive for the protozoan were carrying. While this was somewhat circumvented by combining the Blastocystis STs to analyse the protozoan at the genus level, the findings therefore lack specificity.

A further limitation is that PCR testing only indicates the presence of protozoa, but does not provide information on whether the organisms are dead or alive (Cangelosi \& Meschke, 2014). Therefore, it is possible that while individuals may have tested positive for Blastocystis sp. or D. fragilis, these organisms may have been dead and therefore not exerting their typical influence. Despite this, PCR testing is thought to be best practice for detecting intestinal protozoa (De Canale et al., 2009; Garcia et al., 2018; Padukone et al., 2018). Roberts et al. (2011) suggest that the results of studies that rely on microscopy alone should be interpreted with caution. It may be that differences in diagnostic techniques contribute to the controversy surrounding the relationship between intestinal protozoa and symptomatology due to the underestimation of intestinal protozoa.

Despite these limitations, the current study is the first to investigate differences in psychological symptom expression related to Blastocysitis and/or D. fragilis carriage, acting as an important first step for further research and elucidation of the possible influence of these protozoa on the holistic well-being of their human host. Future studies must make a concerted effort to address the aforementioned limitations. While it may currently be impossible to investigate the intricate multi-directional relationships within and beyond the gut, research in this area should endeavour to take the most holistic possible approach. For example, further studies should consider the possible moderating effects of specific bacteria on the relationship between protozoa and symptom expression. The possible moderating effect of protozoa on the relationship between bacteria and symptoms should also be considered.

\section{Conclusion}

The results of the current study add weight to the argument that Blastocystis sp. and D. fragilis are, potentially, commensal members of the human gut microbiota, and do not appear to contribute to self-reported psychological symptom severity. However, as this is the first study investing psychological symptoms, further research is required before solid conclusions can be drawn regarding the influence of these protozoa on psychological symptoms. Clinical trials investigating specific protozoan subtypes and multiple stool sampling to avoid underestimation of protozoan prevalence is warranted due to continued contradictory findings in relation to these protozoa and other symptom expressions (i.e. GI symptoms). Greater 
research focus on these protozoa is required due to the tremendous inter- and intra-individual differences which may contribute to potential commensalism, symbiosis, or pathogenicity. With further research specifically focusing on the potential mechanisms of action of these protozoa, researchers will gain greater insight into, and understanding of, the variability in symptom expression of protozoan carriers. While the results of this study do not suggest that Blastocystis and D. fragilis should be used in the treatment of various symptomatologies, they do suggest that treatment of asymptomatic carriers of these protozoa should be cautiously considered, given the potential deleterious side effects of such treatments. The role that Blastocystis sp. and D. fragilis play in symptom expression has received comparatively less attention to the bacterial component of the gut microbiota, especially in regard to psychological symptom expression. As such, this paper serves as a call to action for BGMA researchers to consider protozoan members of the gut microbiota going forward to fill this glaring gap in the literature.

Supplementary Information The online version contains supplementary material available at https://doi.org/10.1007/s12144-021-01700-z.

Code Availability Not applicable.

Funding This work was supported by a postgraduate scholarship with no restrictions on publications cofounded by Bioscreen, an industry partner of Victoria University.

Data Availability The data that support the findings of this study are available from Bioscreen but restrictions apply to the availability of these data, which were used under licence for the current study, and so are not publicly available. Data are however available from the authors upon reasonable request and with permission of Bioscreen.

\section{Declarations}

Conflict of Interest The authors report no conflict of interest.

Open Access This article is licensed under a Creative Commons Attribution 4.0 International License, which permits use, sharing, adaptation, distribution and reproduction in any medium or format, as long as you give appropriate credit to the original author(s) and the source, provide a link to the Creative Commons licence, and indicate if changes were made. The images or other third party material in this article are included in the article's Creative Commons licence, unless indicated otherwise in a credit line to the material. If material is not included in the article's Creative Commons licence and your intended use is not permitted by statutory regulation or exceeds the permitted use, you will need to obtain permission directly from the copyright holder. To view a copy of this licence, visit http://creativecommons.org/licenses/by/4.0/.

\section{References}

Abu-Madi, M. A., Behnke, J. M., Boughattas, S., Al-Thani, A., \& Doiphode, S. H. (2016). A decade of intestinal protozoan epidemiology among settled immigrants in Qatar. BMC Infectious Diseases, 16, 370-370. https://doi.org/10.1186/s12879-016-1728-3.

Akoglu, H. (2018). User's guide to correlation coefficients. Turkish Journal of Emergency Medicine, 18, 91-93. https://doi.org/10. 1016/j.tjem.2018.08.001.

Al-Hindi, A., \& Shammala, B. M. A. (2013). Dientamoeba fragilis in Gaza Strip: a neglected protozoan parasite. Iranian Journal of Parasitology, 8(2), 249-255.

Altemus, M., Sarvaiya, N., \& Neill Epperson, C. (2014). Sex differences in anxiety and depression clinical perspectives. Frontiers in Neuroendocrinology, 35(3), 320-330. https://doi.org/10.1016/j. yfrne.2014.05.004.

Audebert, C., Even, G., Cian, A., El Safadi, D., Certad, G., Delhaes, L., Pereira, B., Nourrisson, C., Poirier, P., Wawrzyniak, I., Delbac, F., Morelle, C., Bastien, P., Lachaud, L., Bellanger, A., Botterel, F., Candolfi, E., Desoubeaux, G., Morio, F., Pomares, C., Rabodonirina, M., Loywick, A., Merlin, S., Viscogliosi, E., \& Chabé, M. (2016). Colonization with the enteric protozoa Blastocystis is associated with increased diversity of human gut bacterial microbiota. Scientific Reports, 6, 25255. https://doi.org/ $10.1038 /$ srep25255.

Aykur, M., Caliskan Kurt, C., Dirim Erdogan, D., Biray Avci, C., Vardar, R., Aydemir, S., Girginkardeșler, N., Gündüz, C., \& Dagci, H. (2019). Investigation of Dientamoeba fragilis prevalence and evaluation of Sociodemographic and clinical features in patients with gastrointestinal symptoms. Acta Parasitologica, 64(1), 162-170. https://doi.org/10.2478/s11686-018-00017-5.

Barratt, J. L., Harkness, J., Marriott, D., Ellis, J. T., \& Stark, D. (2011). A review of Dientamoeba fragilis carriage in humans: Several reasons why this organism should be considered in the diagnosis of gastrointestinal illness. Gut Microbes, 2(1), 3-12. https://doi.org/10.4161/ gmic.2.1.14755.

Barsky, A. J., Peekna, H. M., \& Borus, J. F. (2001). Somatic symptom reporting in women and men. Journal of General Internal Medicine, 16(4), 266-275.

Bart, A., Wentink-Bonnema, E. M., Gilis, H., Verhaar, N., Wassenaar, C. J., van Vugt, M., Goorhuis, A., \& van Gool, T. (2013). Diagnosis and subtype analysis of Blastocystis sp. in 442 patients in a hospital setting in the Netherlands. BMC Infectious Diseases, 13, 389. https://doi.org/10.1186/1471-2334-13-389.

Beasley, T. M., \& Schumacker, R. E. (1995). Multiple regression approach to analyzing contingency tables: Post hoc and planned comparison procedures. The Journal of Experimental Education, 64(1), 79-93. https://doi.org/10.1080/00220973.1995.9943797.

Beghini, F., Pasolli, E., Truong, T. D., Putignani, L., Cacciò, S. M., \& Segata, N. (2017). Large-scale comparative metagenomics of Blastocystis, a common member of the human gut microbiome. The ISME Journal, 11, 2848-2863. https://doi.org/10.1038/ismej. 2017.139.

Beiromvand, M., Hashemi, S. J., Arjmand, R., Sadjadei, N., \& Hardanipasand, L. (2017). Comparative prevalence of Blastocystis in patients with the irritable bowel syndrome and healthy individuals: A case control study. Jundishapur Journal of Microbiology, 10(6), e13572. https://doi.org/10.5812/jjm.13572. 
Boccabella, A., \& Malouf, J. (2017). How do sleep-related health problems affect functional status according to sex? Journal of Clinical Sleep Medicine, 13(5), 685-692. https://doi.org/10.5664/jcsm.6584.

Brands, M. R., Van de Vijver, E., Haisma, S. M., Heida, A., \& van Rheenen, P. F. (2019). No association between abdominal pain and Dientamoeba in Dutch and Belgian children. Archives of Disease in Childhood, archdischild-2018-316383. https://doi.org/ 10.1136/archdischild-2018-316383.

Bruce-Keller, A. J., Salbaum, J. M., \& Berthoud, H. R. (2018). Harnessing gut microbes for mental health: Getting from Here to there. Biological Psychiatry, 83(3), 214-223. https://doi.org/10. 1016/j.biopsych.2017.08.014.

Burgess, S. L., Gilchrist, C. A., Lynn, T. C., \& Petri, W. A. (2017). Parasitic Protozoa and interactions with the host intestinal microbiota. Infection and Immunity, 85(8), e00101-e00117. https://doi.org/ 10.1128/IAI.00101-17.

Butler, W. P. (1996). Dientamoeba fragilis. An unusual intestinal pathogen. Digestive Diseases and Sciences, 41(9), 1811-1813. https:// doi.org/10.1007/bf02088750.

Cangelosi, G. A., \& Meschke, J. S. (2014). Dead or alive: Molecular assessment of microbial viability. Applied and Environmental Microbiology, 80(19), 5884-5891. https://doi.org/10.1128/aem. 01763-14.

Chabé, M., Lokmer, A., \& Segurel, L. (2017). Gut Protozoa: Friends or foes of the human gut microbiota? Trends in Parasitology, 33(12), 925-934. https://doi.org/10.1016/j.pt.2017.08.005.

Chandramathi, S., Suresh, K., Anita, Z. B., \& Kuppusamy, U. R. (2012). Infections of Blastocystis hominis and microsporidia in cancer patients: Are they opportunistic? Transactions of the Royal Society of Tropical Medicine and Hygiene, 106(4), 267-269. https://doi.org/ 10.1016/j.trstmh.2011.12.008.

Chandramathi, S., Suresh, K., Sivanandam, S., \& Kuppusamy, U. R. (2014). Stress exacerbates infectivity and pathogenicity of Blastocystis hominis: In vitro and in vivo evidences. PLoS One, 9(5), e94567-e94567. https://doi.org/10.1371/journal.pone. 0094567.

Chudnovskiy, A., Mortha, A., Kana, V., Kennard, A., Ramirez, J. D., Rahman, A., Remark, R., Mogno, I., Ng, R., Gnjatic, S., Amir, E. A. D., Solovyov, A., Greenbaum, B., Clemente, J., Faith, J., Belkaid, Y., Grigg, M. E., \& Merad, M. (2016). Host-protozoan interactions protect from mucosal infections through activation of the inflammasome. Cell, 167(2), 444-456. https://doi.org/10.1016/j. cell.2016.08.076.

Cohen, J. (1969). Statistical power analysis for the behavioural sciences. Academic Press.

Cohen, J., Cohen, P., West, S. G., \& Aiken, L. S. (2013). Applied multiple regression/correlation analysis for the behavioural sciences (3rd ed.). Lawrence Erlbaum Associates Publishers.

Coulson, S., Butt, H., Vecchio, P., Gramotnev, H., \& Vietta, L. (2013). Green-lipped mussel extract (Perna canaliculus) and glucosamine sulphate in patients with knee osteoarthritis: Therapeutic efficacy and effects on gastrointestinal microbiota profiles. Inflammopharmacology, 21, 79-90.

Coyle, C. M., Varughese, J., Weiss, L. M., \& Tanowitz, H. B. (2011). Blastocystis: To treat or not to treat.... Clinical Infectious Diseases, 54(1), 105-110. https://doi.org/10.1093/cid/cir810.

Crotti, D., \& D'Annibale, M. L. (2007). Human intestinal parasitosis: Role of Dientamoeba fragilis in human infections. Annali di Igiene, 19(1), 27-34.
Dawkins, R., \& Krebs, J. R. (1979). Arms race between and within species. Proceedings of the Royal Society of London. Series B, Biological Sciences, 205(1161), 489-511.

De Canale, E., Biasolo, M. A., Tessari, A., Bettanello, S., Besutti, V., \& Mengoli, C. (2009). Real time PCR for Dientamoeba fragilis: A comparison between molecular and microscopical approach. Microbiologia Medica, 24(3). https://doi.org/10.4081/mm.2009. 2524.

Deng, L., Wojciech, L., Gascoigne, N. R. J., Peng, G., \& Tan, K. S. W. (2021). New insights into the interactions between Blastocystis, the gut microbiota, and host immunity. PLoS Pathogens, 17(2), e1009253. https://doi.org/10.1371/journal.ppat.1009253.

Dunwell, D. (2013). ME/CFS and Blastocystis spp or Dientamoeba fragilis, an in-house comparison. British Journal of General Practice, 63(607), 73-74. https://doi.org/10.3399/bjgp13X663028.

Durk, R. P., Castillo, E., Márquez-Magaña, L., Grosicki, G. J., Bolter, N. D., Lee, C. M., \& Bagley, J. R. (2019). International Journal of Sport Nutrition and Exercise Metabolism, 29(3), 249-253. https:// doi.org/10.1123/ijsnem.2018-0024.

El Aidy, S., Stilling, R., Dinan, T. G., \& Cryan, J. F. (2016). Microbiome to brain: Unravelling the multidirectional axes of communication. Advances in Experimental Medicine and Biology, 874, 301-336. https://doi.org/10.1007/978-3-319-20215-0_15.

El-Zawawy, H. T., Farag, H. F., Tolba, M. M., \& Abdalsamea, H. A. (2020). Improving Hashimoto's thyroiditis by eradicating Blastocyistis hominis: Relation to IL-17. Theraputic Advances in Endocrinology and Metabolism, 11, 1-8. https://doi.org/10.1177/ 2042018820907013.

Flak, M. B., Neves, J. F., \& Blumberg, R. S. (2013). Immunology. Welcome to the microgenderome. Science, 339(6123), 10441045. https://doi.org/10.1126/science.1236226.

Garcia, L. S. (2016). Dientamoeba fragilis; one of the neglected intestinal Protozoa. Journal of Clinical Microbiology, 54(9), 2243-2250. https://doi.org/10.1128/JCM.00400-16.

Garcia, L. S., Arrowood, M., Kokoskin, E., Paltridge, G. P., Pillai, D. R., Procop, G. W., Ryan, N., Shimizu, R. Y., \& Visvesvara, G. (2018). Laboratory diagnosis of parasites from the gastrointestinal tract. Clinical Microbiology Reviews, 31(1), e00025-e00017. https://doi. org/10.1128/CMR.00025-17.

Giloteaux, L., Goodrich, J. K., Walters, W. A., Levine, S. M., Ley, R. E., \& Hanson, M. R. (2016). Reduced diversity and altered composition of the gut microbiome in individuals with myalgic encephalomyelitis/chronic fatigue syndrome. Microbiome, 4(1), 30. https://doi.org/10.1186/s40168-016-0171-4.

Grendon, J. H., DiGiacomo, R. F., \& Frost, F. J. (1995). Descriptive features of Dientamoeba fragilis infections. The Journal of Tropical Medicine and Hygiene, 98(5), 309-315.

Hall, E., \& Steiner, M. (2013). Serotonin and female psychopathology. Women's Health, 9(1), 85-97. https://doi.org/10.2217/WHE.12.64.

Hamidi, N., Meamar, A. R., Akhlaghi, L., Rampisheh, Z., \& Razmjou, E. (2018). Dientamoeba fragilis diagnosis be fecal screening: Relative effectiveness of traditional techniques and molecular methods. The Journal of Infection in Developing Countries, 12(1), 52-59. https:// doi.org/10.3855/jidc. 9643 .

Hayes, A. F. (2017). Introduction to mediation, moderation, and conditional process analysis: A regression-based approach. Guilford publication.

Ho, J. M. W., \& Juurlink, D. N. (2011). Considerations when prescribing trimethoprim-sulfamethoxazole. Canadian Medical Association 
Journal, 183(16), 1851-1858. https://doi.org/10.1503/cmaj. 111152.

Holtman, G. A., Kranenberg, J. J., Blanker, M. H., Ott, A., Lisman-van Leeuwen, Y., \& Berger, M. Y. (2017). Dientamoeba fragilis colonization is not associated with gastrointestinal symptoms in children at primary care level. Family Practice, 34(1), 25-29. https://doi.org/ 10.1093/fampra/cmw111.

Huberty, C. J, \& Petoskey, M. D. (2000). Multivariate analysis of variance and covariance. In H. E. A. Tinsley \& S. D. Brown (Eds.), Handbook of applied multivariate statistics and mathematical modeling (p. 183-208). Academic Press.https://doi.org/10.1016/ B978-012691360-6/50008-2

Jiang, H., Ling, Z., Zhang, Y., Mao, H., Ma, Z., Yin, Y., Wang, W., Tang, W., Tan, Z., Shi, J., Li, L., \& Ruan, B. (2015). Altered fecal microbiota composition in patients with major depressive disorder. Brain, Behaviour, and Immunity, 48, 186-194. https://doi.org/10.1016/j. bbi.2015.03.016.

Jiménez, P. A., Jaimes, J. E., \& Ramírez, J. D. (2019). A summary of Blastocystis subtypes in north and South America. Parasites \& Vectors, 12(1), 376. https://doi.org/10.1186/s13071-019-3641-2.

Johnson, E. H., Windsor, J. J., \& Clark, C. G. (2004). Emerging from obscurity: Biological, clinical, and diagnostic aspects of Dientamoeba fragilis. Clinical Microbiology Review, 17(3), 553570. https://doi.org/10.1128/CMR.17.3.553-570.2004.

Jokelainen, P., Hebbelstrup Jensen, B., Andreassen, B. U., Petersen, A. M., Roser, D., Krogfelt, K. A., Nielsen, H. V., \& Stensvold, C. R. (2017). Dientamoeba fragilis, a commensal in children in Danish day care centers. Journal of Clinical Microbiology, 55(6), 17071713. https://doi.org/10.1128/jcm.00037-17.

Kaneda, Y., Horiki, N., Cheng, X., Tachibana, H., \& Tsutsumi, Y. (2000). Serologic response to Blastocystis hominis infection in asymptomatic individuals. The Tokai Journal of Experimental and Clinical Medicine, 25(2), 51-56.

Kesuma, Y., Firmansyah, A., Bardosono, S., Sari, I. P., \& Kurniawan, A. (2019). Blastocystis ST-1 is associated with irritable bowel sundrom-diarrhoea (ISB-D) in Indonesian adolescence. Parasite Epidemiology and Control, 6, 1-9. https://doi.org/10.1016/j.parepi. 2009.e00112.

Klein, S. L. (2004). Hormonal and immunological mechanisms mediating sex differences in parasite infection. Parasite Immunology, 26(6-7), 247-264. https://doi.org/10.1111/j.0141-9838.2004. 00710.x.

Kovats, S. (2015). Estrogen receptors regulate innate immune cells and signaling pathways. Cellular Immunology, 294(2), 63-69. https:// doi.org/10.1016/j.cellimm.2015.01.018.

Krogsgaard, L. R., Engsbro, A. L., Stensvold, C. R., Nielsen, H. V., \& Bytzer, P. (2015). The prevalence of intestinal parasites is not greater among individuals with irritable bowel syndrome: A populationbased case-control study. Clinical Gastroenterology and Hepatology, 13(3), 507-513.e502. https://doi.org/10.1016/j.cgh. 2014.07.065

Krogsgaard, L. R., Andersen, L. O. B., Johannesen, T. B., Engsbro, A. L., Stensvold, C. R., Nielsen, H. V., \& Bytzer, P. (2018). Characteristics of the bacterial microbiome in association with common intestinal parasites in irritable bowel syndrome. Clinical and Translational Gastroenterology, 9(6), 161-161. https://doi.org/10. 1038/s41424-018-0027-2.

Laanto, E., Hoikkala, V., Ravantti, J., \& Sundberg, L. R. (2017). Longterm genomic coevolution of host-parasite interaction in the natural environment. Nature Communications, 8, 1-8. https://doi.org/10. 1038/s41467-017-00158-7.

Lepczyńska, M., Białkowska, J., Dzika, E., Piskorz-Ogórek, K., \& Korycińska, J. (2017). Blastocystis: How do specific diets and human gut microbiota affect its development and pathogenicity? European journal of clinical microbiology \& infectious diseases: official publication of the European Society of Clinical Microbiology, 36(9), 1531-1540. https://doi.org/10.1007/s10096017-2965-0.

Leung, J. M., Graham, A. L., \& Knowles, S. C. (2018). Parasitemicrobiota interaction with the vertebrate gut: Synthesis through an ecological lens. Frontiers in Microbiology, 9(843), 1-20. https://doi.org/10.3389/fmic.2018.00843.

Li, Q., Han, Y., Dy, A. B. C., \& Hagerman, R. J. (2017). The gut microbiota and autism Spectrum disorders. Frontiers in Cellular Neuroscience, 11, 120-120. https://doi.org/10.3389/fncel.2017. 00120.

Lloyd-Price, J., Abu-Ali, G., \& Huttenhower, C. (2016). The healthy human microbiome. Genome Medicine, 8(1), 51. https://doi.org/ 10.1186/s13073-016-0307-y.

Lokmer, A., Cian, A., Froment, A., Gantois, N., Viscogliosi, E., Chabé, M., \& Ségurel, L. (2019). Use of shotgun metagenomics for the identification of protozoa in the gut microbiota of healthy individuals from worldwide populations with various industrialization levels. PLoS One, 14(2), e0211139. https://doi.org/10.1371/ journal.pone.0211139.

Lukeš, J., Stensvold, C. R., Jirků-Pomajbíková, K., \& Wegener Parfrey, L. (2015). Are human intestinal eukaryotes beneficial or commensals? PLoS Pathogens, 11(8), e1005039. https://doi.org/10.1371/ journal.ppat.1005039.

MacLean, A., Sweeting, H., \& Hunt, K. (2010). 'Rules' for boys, 'guidelines' for girls: Gender differences in symptom reporting during childhood and adolescence. Social Science and Medicine, 70(4), 597-604. https://doi.org/10.1016/j.socschimedi.2009.10.042.

Maeng, L. Y., \& Milad, M. R. (2015). Sex differences in anxiety disorders: Interactions between fear, stress, and gonadal hormones. Hormones and Behaviour, 76, 106-117. https://doi.org/10.1016/j. yhbeh.2015.04.002.

Maizels, R. M. (2009). Parasite immunomodulation and polymorphisms of the immune system. Journal of Biology, 8(62), 1-4. https://doi. org/10.1186/jbioll66.

Markle, J. G., Frank, D. N., Mortin-Toth, S., Robertson, C. E., Feazel, L. M., Rolle-Kampczyk, U., von Bergen, M., McCoy, K. D., Macpherson, A. J., \& Danska, J. S. (2013). Sex differences in the gut microbiome drive hormone-dependent regulation of autoimmunity. Science, 339(6123), 1084-1088. https://doi.org/10.1126/ science. 1233521.

Marriott, I., \& Huet-Hudson, Y. M. (2006). Sexual dimorphism in innate immune responses to infectious organisms. Immunological Research, 34(3), 177-192. https://doi.org/10.1385/ir:34:3:177.

McHugh, M. L. (2013). The chi-square test of independence. Biochemica Medica, 23(2), 143-149. https://doi.org/10.11613/BM.2013.018.

Menendez Fernandez-Miranda, C., Fernandez-Suarez, J., RodriguezPerez, M., Menendez Fernandez-Miranda, P., Vazquez, F., Boga Ribeiro, J. A., \& Rodriguez-Guardado, A. (2018). Prevalence of D. fragilis infection in the household contacts of a group of infected patients. Enfermedades Infecciosas y Microbiología Clínica, 36(7), 423-427. https://doi.org/10.1016/j.eimc.2017.08.004. 
Mulak, A., Taché, Y., \& Larauche, M. (2014). Sex hormones in the modulation of irritable bowel syndrome. World Journal of Gastroenterology, 20(10), 2433-2448. https://doi.org/10.3748/wjg. v20.i10.2433.

Nagata, N., Marriott, D., Harkness, J., Ellis, J. T., \& Start, D. (2012). Current treatment options for Dientamoeba fragilis infections. The International Journal for Parasitology - Drugs and Drug Resistance, 2, 204-215. https://doi.org/10.1016/j.ijpddr.2012.08. 002 .

Norberg, A., Nord, C. E., \& Evengard, B. (2003). Dientamoeba fragilis-a protozoal infection which may cause severe bowel distress. Clinical Microbiology and Infection, 9(1), 65-68.

Nourrisson, C., Scanzi, J., Pereira, B., NkoudMongo, C., Wawrzyniak, I., Cian, A., Viscogliosi, E., Livrelli, V., Delbec, F., Dapoigny, M., \& Poirier, P. (2014). Blastocystis is associated with decrease of fecal microbiota protective bacteria: Comparative analysis between patients with irritable bowel syndrome and control subjects. PLoS One, 9(11), e111868. https://doi.org/10.1371/journal.pone. 0111868 .

Padukone, S., Mandal, J., Rajkumari, N., Bhat, B., Swaminathan, R., \& Parija, S. (2018). Detection of Blastocystis in clinical stool specimens using three different methods and morphological examination in Jones' medium. Tropical Parasitology, 8(1), 33-40. https://doi. org/10.4103/tp.TP_4_18.

Pavanelli, M. F., Kaneshima, E. N., Uda, C. F., Colli, C. M., FalavignaGuilherm, A. L., \& Gomes, M. L. (2015). Pathogenicity of Blastocystis sp. to the gastrointestinal tract of mice: Relationship between inoculum size and period of infection. Revista do Instituto de Medicina Tropical de Sao Paulo, 57(6), 467-472. https://doi.org/ 10.1590/S0036-46652015000600002.

Peek, R., Reedeker, F. R., \& van Gool, T. (2004). Direct amplification and genotyping of Dientamoeba fragilis from human stool specimens. Journal of Clinical Microbiology, 42(2), 631-635. https:// doi.org/10.1128/jcm.42.2.631-635.2004.

Qadri, S. M., al-Okaili, G. A., \& al-Dayel, F. (1989). Clinical significance of Blastocystis hominis. Journal of Clinical Microbiology, 27(11), 2407-2409.

Rea, K., Dinan, T. G., \& Cryan, J. F. (2016). The microbiome: A key regulator of stress and neuroinflammation. Neurobiology of Stress, 4, 23-33. https://doi.org/10.1016/j.ynstr.2016.03.001.

Rhoads, S. E., \& Rhoads, C. H. (2012). Gender roles and infant/toddler care: Male and female professors on the tenure track. Journal of Social, Evolutionary, and Cultural Psychology, 6(1), 13-31. https://doi.org/10.1037/h0099227.

Richardson, J. T. E. (2011). Eta squared and partial eta squared as measures of effect size in educational research. Educational Research Review, 6(2), 135-147. https://doi.org/10.1016/j.edurev.2010.12. 001

Rinninella, E., Raoul, P., Cintoni, M., Franceschi, F., Miggiano, G. A. D., Gasbarrini, A., \& Mele, M. C. (2019). What is the healthy gut microbiota composition? A changing ecosystem across age, environment, diet, and diseases. Microorganisms, 7(1), 14. https://doi. org/10.3390/microorganisms7010014.

Roberts, T., Barratt, J., Harkness, J., Ellis, J., \& Stark, D. (2011). Comparison of microscopy, culture, and conventional polymerase chain reaction for detection of blastocystis sp. in clinical stool samples. The American Journal of Tropical Medicine and Hygiene, 84(2), 308-312. https://doi.org/10.4269/ajtmh.2011.10-0447.
Rook, G. A. (2013). Regulation of the immune system by biodiversity from the natural environment: An ecosystem service essential to health. Proceedings of the National Academy of Sciences of the United States of America, 110(46), 18360-18367. https://doi.org/ 10.1073/pnas.1313731110.

Rook, G. A., \& Brunet, L. R. (2005). Microbes, immunoregulation, and the gut. Gut, 54(3), 317-320. https://doi.org/10.1136/gut.2004. 053785.

Rook, G. A., Raison, C. L., \& Lowry, C. A. (2014). Microbial 'old friends', immunoregulation and socioeconomic status. Clinical and Experimental Immunology, 177(1), 1-12. https://doi.org/10.1111/ cei.12269.

Röser, D., Simonsen, J., Nielsen, H. V., Stensvold, C. R., \& Mølbak, K. (2013). Dientamoeba fragilis in Denmark: Epidemiological experience derived from four years of routine real-time PCR. European Journal of Clinical Microbiology \& Infectious Diseases, 32(10), 1303-1310. https://doi.org/10.1007/s10096-013-1880-2.

Rossen, N. G., Bart, A., Verhaar, N., van Nood, E., Kootte, R., de Groot, P. F., D’Haens, G. R., Ponsioen, C. Y., \& van Gool, T. (2015). Low prevalence of Blastocystis sp. in active ulcerative colitis patients. European journal of clinical microbiology \& infectious diseases: official publication of the European Society of Clinical Microbiology, 34(5), 1039-1044. https://doi.org/10.1007/s10096015-2312-2.

Salehi, M., Mardaneh, J., Niazkar, H. R., Minooeianhaghighi, M., Arshad, E., Soleimani, F., \& Mohammadzadeh, A. (2021). Prevalence and subtype analysis of Blastocystis hominis isolated from patients in the northeast of Iran. Journal of Parasitology Research, 2021, 1-8. https://doi.org/10.1155/2021/8821885.

Scanlan, P. D., Stensvold, C. R., Rajilić-Stojanović, M., Heilig, H. G. H. J., De Vos, W. M., O'Toole, P. W., \& Cotter, P. D. (2014). The microbial eukaryote Blastocystis is a prevalent and diverse member of the healthy human gut microbiota. FEMS Microbiology Ecology, 90(1), 326-330. https://doi.org/10.1111/1574-6941.12396.

Seney, M. L., \& Sibille, E. (2014). Sex differences in mood disorders: Perspectives from humans and rodent models. Biology of Sex Differences, 5(1), 17-17. https://doi.org/10.1186/s13293-0140017-3.

Seyer, A., Karasartova, D., Ruh, E., Güreser, A. S., Turgal, E., Imir, T., \& Taylan-Ozkan, A. (2017). Epidemiology and prevalence of Blastocystis spp. in North Cyprus. The American Journal of Tropical Medicine and Hygiene, 96(5), 1164-1170. https://doi.org/ 10.4269/ajtmh.16-0706.

Solomon, M. B., \& Herman, J. P. (2009). Sex differences in psychopathology: Of gonads, adrenals and mental illness. Physiology \& Behaviour, 97(2), 250-258. https://doi.org/10.1016/j.physbeh. 2009.02.033.

Sorge, R. E., \& Strath, L. J. (2018). Sex differences in pain responses. Current Opinion in Physiology, 6, 75-81. https://doi.org/10.1016/j. cophys.2018.05.006.

Sorge, R. E., \& Totsch, S. K. (2017). Sex differences in pain. Journal of Neuroscience Research, 95(6), 1271-1281. https://doi.org/10.1002/ jnr.23841.

Stark, D., Barratt, J., Roberts, T., Marriott, D., Harkness, J., \& Ellis, J. (2010). A review of the clinical presentation of dientamoebiasis. The American Journal of Tropical Medicine and Hygiene, 82(4), 614 619. https://doi.org/10.4269/ajtmh.2010.09-0478.

Stark, D., Barratt, J., Chan, D., \& Ellis, J. T. (2016). Dientamoeba fragilis, the neglected trichomonad of the human bowel. Clinical 
Microbiology Reviews, 29(3), 553-580. https://doi.org/10.1128/ CMR.00076-15.

Stensvold, C. R., \& Clark, C. G. (2016). Current status of Blastocystis: A personal view. Parasitol Int, 65(6 Pt B), 763-771. https://doi.org/10. 1016/j.parint.2016.05.015.

Stojanov, S., Berlec, A., \& Štrukelj, B. (2020). The influence of probiotics on the Firmicutes/Bacteroidetes ratio in the treatment of obesity and inflammatory bowel disease. Microorganisms, 8(1715), 1-16. https://doi.org/10.3390/microorganisms 8111715 .

Taneja, V. (2018). Sex Hormones Determine Immune Response. Frontiers in Immunology, 9, 1931-1931. https://doi.org/10.3389/ fimmu.2018.01931.

Tito, R. Y., Chaffron, S., Caenepeel, C., Lima-Mendez, G., Wang, J., Vieira-Silva, S., Falony, G., Hildebrand, F., Darzi, Y., Rymenans, L., Verspecht, C., Bork, P., Vermeire, S., Joossens, M., \& Raes, J. (2019). Population-level analysis of Blastocystis subtype prevalence and variation in the human gut microbiota. Gut, 68(7), 1180-1189. https://doi.org/10.1136/gutjnl-2018-316106.

Tsaousis, A. D., Hamblin, K. A., Elliott, C. R., Young, L., RosellHidalgo, A., Gourlay, C. W., Moore, A. L., \& van der Giezen, M. (2018). The human gut colonizer Blastocystis respires using complex II and alternative oxidase to buffer transient oxygen fluctuations in the gut. Frontiers in Cellular and Infection Microbiology, 8, 371-371. https://doi.org/10.3389/fcimb.2018.00371.

Vaiserman, A., Romanenko, M., Piven, L., Moseiko, V., Lushchak, O., Kryzhanovska, N., Guryanov, V., \& Koliada, A. (2020). Differences in the gut Firmicutes and Bacteroidetes ratio across age groups in healthy Ukrainian population. BMC Microbiology, 20, 1-8. https://doi.org/10.1186/s12866-020-01903-7.

Valdes, A. M., Walter, J., Segal, E., \& Spector, T. D. (2018). Role of the gut microbiota in nutrition and health. $B M J, 361, \mathrm{k} 2179$. https://doi. org/10.1136/bmj.k2179.

van Polanen, M., Colonnesi, C., Tavecchio, L. W. C., Blokhuis, S., \& Fukkink, R. G. (2017). Men and women in childcare: A study of caregiver-child interactions. European Early Childhood Education Research Journal, 25(3), 412-424. https://doi.org/10.1080/ 1350293X.2017.1308165.

Vemuri, R., Sylvia, K. E., Klein, S. L., Forster, S. C., Plebanski, M., Eri, R., \& Flanagan, K. L. (2019). The microgenderome revealed: Sex differences in bidirectional interactions between the microbiota, hormones, immunity and disease susceptibility. Seminars in Immunopathology, 41(2), 265-275. https://doi.org/10.1007/ s00281-018-0716-7.

Vogt, N. M., Kerby, R. L., Dill-McFarland, K. A., Harding, S. J., Merluzzi, A. P., Johnson, S. C., Carlsson, C. M., Asthana, S., Zetterberg, H., Blennow, K., Bendlin, B. B., \& Rey, F. E. (2017). Gut microbiome alterations in Alzheimer's disease. Scientific Reports, 7(1), 13537. https://doi.org/10.1038/s41598-017-13601-y.

Wallis, A., Butt, H., Ball, M., Lewis, D. P., \& Bruck, D. (2016). Support for the Microgenderome: Associations in a human clinical population. Scientific Reports, 6, 19171. https://doi.org/10.1038/ srep19171.

Weir, C. B. \& Le, J. K. (2020). Metronidazole. In: StatPearls (internet). StatPearls Publishing. Available from: https://www.ncbi.nlm.nih. gov/books/NBK539728/

Yadav, R. K., Malhotra, S., \& Duggal, N. (2020). Immune dysfunction during enteric protozoal infection: The current trends. In S. K. Saxena \& H. Prakash Innate Immunity in Health and Disease. IntechOpen. https://doi.org/10.5772/intechopen.91698.

Zuk, M. (2009). The Sicker Sex. PLOS Pathogens, 5(1), e1000267. https://doi.org/10.1371/journal.ppat.1000267.

Zulfa, F., Sari, I. P., \& Kurniawan, A. (2017). Association of Blastocystis subtypes with diarrhea in children. Journal of Physics: Conference Series, 884, 1-6.

Publisher's Note Springer Nature remains neutral with regard to jurisdictional claims in published maps and institutional affiliations. 